presenting with the four characteristic symptoms (finger agnosia, agraphia, right-left disorientation and dyscalculia), in the absence of any other neuropsychological disorders, had a focal ischemic lesion in the inferior left angular gyrus, extending to the callosal fibers. An impairment in mental manipulation of images and visuospatial function is the basis for the Gerstmann cognitive deficit syndrome.

\title{
PREFRONTAL DYSFUNCTION IN ADHD: FUNCTIONAL MRI STUDY
}

Functional magnetic resonance imaging (MRI), during performance of two tasks requiring high-level executive control, was studied in seven adolescent boys with ADHD, and compared to nine controls, at the Institute of Psychiatry, Kings' College, London, UK. A "stop" task required inhibition of a planned motor response, and a "delay" motor timing task required the synchronization of a motor response to an intermittently appearing visual stimulus. Adolescents with ADHD showed less brain activity, predominantly in the right hemisphere mesial frontal cortex during both tasks, and in the right inferior prefrontal cortex and left caudate nucleus during the stop task. ADHD is associated with subnormal activation of the prefrontal areas responsible for higher-order, inhibitory motor control. (Rubia K, Overmeyer S, Taylor E et al. Hypofrontality in attention deficit hyperactivity disorder during higher-order motor control: a study with funcional MRI. Am I Psychiatry June 1999;156:891-896). (Reprints: Dr Rubia, MRC Child and Adolescent Psychiatry Unit, Institute of Psychiatry, De Crespigny Park, London SE5 8AF, UK).

COMMENT. Motor attention and response selection, impaired in patients with $\mathrm{ADHD}$, is associated with hypofunction of the right mesial frontal lobe and striatal areas. The findings in these functional MRI studies corroborate previous documentation by quantitative MRI of structural changes in the frontal lobe and caudate in ADHD children. (see Progress in Pediatric Neurology III, PNB Publ, 1997;p212; and Vol. II, 1994;pp172-184).

The role of the right frontal lobe in humor appreciation is evaluated at the University of Toronto and Rotman Research Institute, Baycrest Centre for Geriatric Care, Canada. (Shammi P, Stuss DT. Brain June 1999;122:657$666)$. In patients with damage to the right frontal lobe, the physical or emotional responses of laughter and smiling were diminished, and impaired performance on humor appreciation tests was correlated with cognitive deficits. Working memory, or the ability to retain information, was related to appreciation of verbal (jokes) and non-verbal (cartoon) tests.

A right frontal lobe dysfunction may explain the anhedonia characteristic of some children with ADHD.

\section{PREVALENCE OF ADHD AND METHYLPHENIDATE USAGE}

An analysis of data obtained from the National Ambulatory Medical Care Survey (NAMCS), 1990-1995, for children aged 5 through 18 years, was performed at Washington State University, to discern trends in the prevalence of US officebased visits for ADHD and for prescriptions of stimulant medications, including methylphenidate (MPH), for ADHD treatment. From approximately 1 million diagnosed cases in 1990, the prevalence of ADHD had increased 2.3-fold in 1995. In this period, the number of office visits for girls diagnosed with ADHD rose 3.9fold; the mean age of patients with ADHD increased by more than 1 year, from 9.7 to 10.8; the percentage of all office visits resulting in a diagnosis of ADHD rose from $1.1 \%$ to $2.8 \%$; and the population-adjusted rate of ADHD patients prescribed 